Copyright (C) 2008 IEEE. Reprinted from IEEE Transactions on Signal Processing, 2007; 55 (8):4091-4103

This material is posted here with permission of the IEEE. Such permission of the IEEE does not in any way imply IEEE endorsement of any of the University of Adelaide's products or services. Internal or personal use of this material is permitted. However, permission to reprint/republish this material for advertising or promotional purposes or for creating new collective works for resale or redistribution must be obtained from the IEEE by writing to pubs-permissions@ieee.org.

By choosing to view this document, you agree to all provisions of the copyright laws protecting it. 


\title{
Optimum Receiver Design for Broadband Doppler Compensation in Multipath/Doppler Channels With Rational Orthogonal Wavelet Signaling
}

\author{
Limin Yu and Langford B. White, Senior Member, IEEE
}

\begin{abstract}
In this paper, we address the issue of signal transmission and Doppler compensation in multipath/Doppler channels. Based on a wavelet-based broadband Doppler compensation structure, this paper presents the design and performance characterization of optimum receivers for this class of communication systems. The wavelet-based Doppler compensation structure takes account of the coexistence of multiple Doppler scales in a multipath/Doppler channel and captures the information carried by multiple scaled replicas of the transmitted signal rather than an estimation of an average Doppler as in conventional Doppler compensation schemes. The transmitted signal is recovered by the perfect reconstruction (PR) wavelet analysis filter bank (FB). We demonstrate that with rational orthogonal wavelet signaling, the proposed communication structure corresponds to a $L$ th-order diversity system, where $L$ is the number of dominant transmission paths. Two receiver designs for pulse amplitude modulation (PAM) signal transmission are presented. Both receiver designs are optimal under the maximum-likelihood (ML) criterion for diversity combination and symbol detection. Good performance is achieved for both receivers in combating the Doppler effect and intersymbol interference (ISI) caused by multipath while mitigating the channel noise. In particular, the second receiver design overcomes symbol timing sensitivities present in the first design at reasonable cost to performance.
\end{abstract}

Index Terms-Doppler compensation, multipath/Doppler channel, optimum receiver, rational orthogonal wavelet, wavelet signaling.

\section{INTRODUCTION}

D OPPLER dispersion arising from the relative motion of transceivers plays a significant role in many communication systems. Combined with multipath channel geometry, it leads to the important characteristics of multipath/Doppler channels as mentioned in [1]: for narrowband signal propagation, a multipath/Doppler channel exhibits 1) rapid changes in signal strength in a small interval, 2) random frequency modulation due to varying Doppler shifts on different multipath signals, and 3) time dispersion caused by multipath propagation delay (frequency-selective fading). In contrast, in the wideband case, the received signal will be distorted but the signal strength does not change rapidly in time. Moreover, the Doppler causes time scaling of the signal rather than the frequency shift in the

Manuscript received November 11, 2005; revised November 5, 2006. The associate editor coordinating the review of this manuscript and approving it for publication was Dr. Timothy N. Davidson.

The authors are with the School of Electrical and Electronic Engineering, The University of Adelaide, SA 5005, Australia (e-mail: liminyu@eleceng.adelaide. edu.au; lang.white@adelaide.edu.au).

Digital Object Identifier 10.1109/TSP.2007.896028 narrowband case. The multipath/Doppler channel therefore exhibits random scaling due to varying Doppler scales on different multipath signals. One example of a multipath/Doppler channel is the underwater acoustic (UWA) channel with mobile transceiver platforms [2].

In the context of UWA communications, many broadband Doppler compensation methods have been developed under a similar structure of time-scaling estimation followed by multirate resampling [2]-[6]. In these cases, Doppler estimation is obtained by transmitting a wideband probe at the start of the data packet. A Doppler preprocessor of the receiver estimates the delay and scale by calculating the ambiguity function of the known probe signal. The residual Doppler is then processed by a subsequent equalizer embedded with the phase-locked loop (PLL). However, for a multipath/Doppler channel, since multiple scales coexist on different multipath signals, the scale estimation followed by resampling structure will inevitably suffer from residual Doppler because an average Doppler scale estimation has to be made before the resampling process.

Addressing this issue, a wavelet-based broadband Doppler compensation structure was proposed in [7]. The proposed structure avoids the explicit estimation of accumulated/instant Doppler scale, that is, the averaging process, by capturing the information carried by signals of all (or dominant) existing Doppler scales and reconstructing the scale-compensated signal via diversity combination. Based on wavelet pulse signaling in the transmitter, a transmultiplexer system model was presented to characterize the multiscale nature of the channel and the recovery of scale-compensated signal based on perfect reconstruction (PR) wavelet filter banks (FBs). The effective implementation of this structure generates new designs of wavelet shaping pulses. A special class of wavelets, rational orthogonal wavelets with a scale dilation factor of $a^{j}: j \in \mathbb{Z}, 1<a<2$ were introduced for this application.

Following from [7], this paper deals with the design and performance characteristics of optimum receivers for this class of wavelet-based communication systems. To be more specific, the presentation of the theory and properties is focused on in this paper for a general class of idealized multipath/Doppler channels as modeled in [2]. The main contributions of this paper are summarized as follows.

1) It gives a further insight into the initial idea of combining rational orthogonal wavelet signaling and PR wavelet FBs for broadband Doppler compensation in multipath/Doppler channels and demonstrates an equivalent system model of an $L$ th-order diversity system. 
2) The paper designs the optimum receivers for the proposed system structure based on the maximum-likelihood (ML) criterion. Two types of receivers are designed. Compared with the type 1 receiver, which is based on traditional optimal correlation/matched filtering demodulators, the type 2 receiver with a modified demodulation structure provides superior robustness against severe time synchronization error. The system performance is examined for the two designed receivers by theoretical derivation of the probability of error in closed form, and supporting Monte Carlo simulations. The tradeoff between system parameters is discussed and evaluated by formulas so that we have full control of the system performance by selecting the parameters to suit specific communication scenarios.

3) The paper provides the groundwork for the design of more frequency-efficient communication systems - the multicarrier modulation (MCM) or the wavelet-based orthogonalfrequency-division-multiplexing (OFDM) communication systems, which utilize rational orthogonal wavelets in different scales as the orthogonal carrier signals.

The paper is organized as follows. To make it a self-contained paper, we provide a review of the wavelet-based Doppler compensation structure in Section II. The design of optimum receivers is detailed in Section III. Section IV presents the simulations that examine the system performance. Conclusions are made in Section V.

\section{RATIONAL WAVELET-BASED TRANSMULTIPLEXER SYSTEM MODEL FOR DOPPLER COMPENSATION}

In this section, we review the broadband Doppler compensation structure with rational wavelet pulse signaling as proposed in [7].

\section{A. Transmultiplexer System Model}

The multipath/Doppler channel is modeled as [2]

$$
y(t)=\sum_{\ell=1}^{L} \int_{-\infty}^{\infty} x\left(\eta_{\ell} t-\tau\right) h_{\ell}\left(\tau-\tau_{\ell}\right) d \tau+\xi(t)
$$

where $L$ is the number of dominant paths, $\eta_{\ell}$ and $\tau_{\ell}$ are the Doppler scale and delay for the path indexed by $\ell, h_{\ell}(t)$ is the impulse response of the channel for path $\ell$ which is defined to be independent of delay and Doppler effects, $x(t)$ and $y(t)$ are the input and output signals, respectively, and $\xi(t)$ is the additive white Gaussian noise (AWGN). We assume flat and independent fading of each path, i.e., $h_{\ell}(t)=\rho_{\ell} \delta(t)$, where $\rho_{\ell}$ is the constant complex attenuation coefficient of path $\ell$ and $\delta(t), t \in \mathbb{R}$ is the Dirac Delta function. Notice that because of the scaling effect by $\eta_{\ell}$, the time delay of each path observed in the receiver is valued at $\tau_{\ell} / \eta_{\ell}$. We take account of this effect and to make the notation simple, $\tau_{\ell}$ is used to denote the delay factor relative to the receiver and is redefined in subsequent equations as $\tau_{\ell}=$ $\tau_{\ell}^{\prime} / \eta_{\ell}$, where $\tau_{\ell}^{\prime}$ is the $\tau_{\ell}$ in (1). The broadband channel model is then simplified to

$$
y(t)=\sum_{\ell=1}^{L} \rho_{\ell} x\left(\eta_{\ell}\left(t-\tau_{\ell}\right)\right)+\xi(t) .
$$

With wavelet pulse signaling, the transmitted signal is expressed as

$$
x(t)=\sum_{k} s(k) \psi(t-k T)
$$

where $\psi(t)$ is the wavelet basis function as the shaping pulse, $T$ is the symbol period, and $\{s(k)\}, k \in \mathbb{Z}$ is the symbol sequence mapped as the amplitude of the shaping pulses. For an $M$-ary-PAM signaling scheme, there is $s(k) \in\left\{s_{m} ; m=\right.$ $1,2, \cdots, M\}$, where $\left\{s_{m}\right\}$ denotes the set of $M$ real equally spaced amplitudes. With the channel model in (2), the received signal can be expressed as

$$
y(t)=\sum_{\ell=1}^{L} \rho_{\ell} \sum_{k} s(k) \psi\left(\eta_{\ell}\left(t-\tau_{\ell}\right)-k T\right)+\xi(t) .
$$

We assume ${ }^{1}$ that the Doppler scales $\left\{\eta_{\ell}, 1 \leq \ell \leq L\right\}$ can be sufficiently approximated by the set $\left\{a^{-\alpha_{\ell}}: a^{-\alpha_{\ell}}=\eta_{\ell}, \alpha_{\ell} \in\right.$ $\mathbb{Z}, 1 \leq \ell \leq L\}$, where $a$ is chosen as $1<a<2, a \in \mathbb{R}$, for a fine partition of the continuous-scale coordinate. We also suppose $y(t)$ is to be sampled with a period of $T_{s}$ in the receiver and the delay $\tau_{\ell}$ can be uniquely decomposed into two parts, $\tau_{\ell}=a^{\alpha_{\ell}} \tau_{\ell}^{(1)} T_{s}+\tau_{\ell}^{(2)} T_{s}$, where $\tau_{\ell}^{(1)}$ and $\tau_{\ell}^{(2)}$ are integers, and $\tau_{\ell}^{(2)} T_{s}<a^{\alpha_{\ell}} T_{s}$. Equation (4) can then be rewritten as

$$
\begin{array}{r}
y(t)=\sum_{\ell=1}^{L} \rho_{\ell} \sum_{n} \sum_{k} s(k) \delta_{d}\left(n-\tau_{\ell}^{(1)}-k T_{n}\right) \psi\left(a^{\alpha_{\ell}} t-n T_{s}\right) \\
* \delta\left(t-\tau_{\ell}^{(2)} T_{s}\right)+\xi(t), \quad n \in \mathbb{Z} \quad \text { (5) }
\end{array}
$$

where $\delta_{d}(n), n \in \mathbb{Z}$ is the Kronecker Delta function, and $T_{n}=$ $\left\lfloor T / T_{s}\right\rfloor$ is the number of samples per symbol where the operation $\lfloor\cdot\rfloor$ rounds $T / T_{s}$ to the nearest integer. The symbol $*$ represents the convolution operation. The delay term $\tau_{\ell}^{(2)}$ can be recognized as the synchronization error and be mitigated via proper tracking techniques in the receiver. Therefore, the term of $\delta\left(t-\tau_{\ell}^{(2)} T_{s}\right)$ in (5) is removed in the subsequent expressions of $y(t)$. Letting $\psi_{k n}(t)=a^{-k / 2} \psi\left(a^{-k} t-n T_{s}\right)$ and using the same notations as in [9], we can then write $y(t)$ as an inverse discrete wavelet transform (DWT)

$$
y(t)=\sum_{\ell=1}^{L} \sum_{n} Y_{\mathrm{DWT}}\left(\alpha_{\ell}, n\right) \psi_{\alpha_{\ell} n}(t)+\xi(t)
$$

where

$$
Y_{\mathrm{DWT}}\left(\alpha_{\ell}, n\right)=a^{\alpha_{\ell} / 2} \rho_{\ell} \sum_{k} s(k) \delta\left(n-\tau_{\ell}^{(1)}-k T_{n}\right)
$$

which is a delayed, attenuated, and periodically zero inserted version of the symbol sequence $\{s(k)\}$. The delay term $\tau_{\ell}^{(1)}$ is

${ }^{1}$ Note that the Doppler scale $\eta_{l}$ can be defined by $\eta_{l}=1+\vartheta_{l}$, where $\vartheta$ is the Doppler shift to carrier frequency ratio [8]. $\vartheta$ is on the order of $10^{-7}$ or less for cellular communications [8] and could reach the order of $10^{-2}$ for vehicular UWA communications [5]. This general assumption holds with a chosen accuracy if $a$ is selected to be sufficiently close to unity. More discussion about the selection of $a$ for practical applications is presented in Section II-B and Section $\mathrm{V}$. 


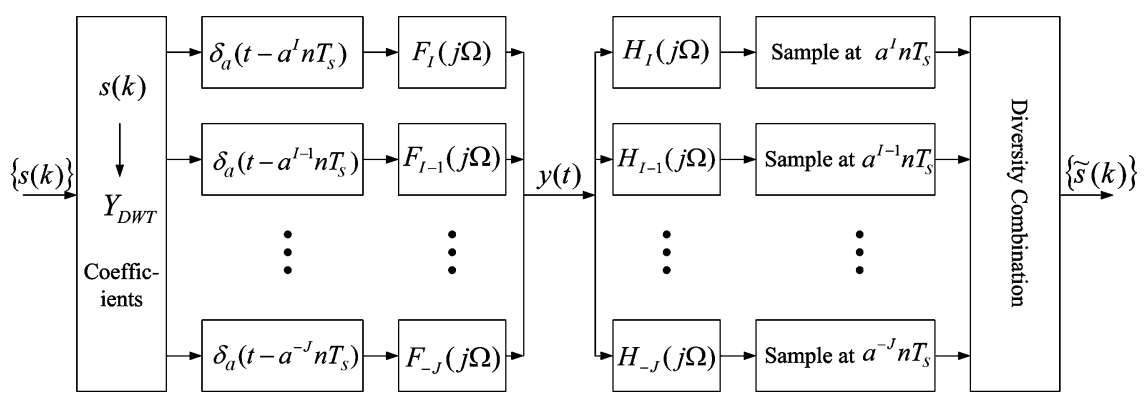

Fig. 1. Transmultiplexer system model.

resolved automatically as a form of delay preceding the wavelet coefficients $Y_{\mathrm{DWT}}\left(\alpha_{\ell}, n\right)$ obtained by DWT.

The whole system can then be modeled as a transmultiplexer FB followed by diversity combination as shown in Fig. 1, where $F_{k}(j \Omega)$ and $H_{k}(j \Omega)$ are transfer functions of wavelet synthesis and analysis filters $f_{k}(t)$ and $h_{k}(t)$ respectively [9]. The filters $f_{k}(t)$ and $h_{k}(t)$ construct a PR wavelet FB and have the relation with the wavelet function $\psi(t)$ as

$$
\begin{aligned}
f(t) & =\psi(t), \\
f_{k}(t) & =a^{-k / 2} f\left(a^{-k} t\right)=\psi_{k}(t)
\end{aligned}
$$

where $f(t)=h^{*}(-t)$ and $f_{k}(t)=h_{k}^{*}(-t)$.

The wavelet signaling and channel transmission are modeled as a wavelet synthesis process. The Doppler dispersion is resolved by wavelet analysis in the receiver with output wavelet coefficients as the multiple versions of the symbol sequence as derived in (7). Notice that this equivalent system model as shown in Fig. 1 resembles a wavelet-based modulation scheme known as the fractal modulation [10]. In other words, the transmission through the multipath/Doppler channel converts a simple PAM signal into a class of rational-homogeneous signals $^{2}$ as in the fractal modulation. Therefore, the receiver part resembles the demodulator of fractal modulation for an ideal AWGN channel. With the knowledge of maximum speed of moving transceivers (either approaching or departing from each other), we can always estimate the maximum Doppler spread and set the reference scales-maximum compression scale $a^{J}$ and maximum dilation scale $a^{-I}$ with $-J \leq \alpha_{\ell} \leq I$, $1<\ell<L, I, J \in \mathbb{Z}^{+}$. Therefore, a maximum of $(I+J+1)$ channels is needed as shown in Fig. 1. The multiple replicas of the transmitted signal carrying the same symbol sequence information can be recovered with PR transmultiplexer FBs.

\section{B. Rational Wavelet Signaling}

As presented in [7], based on the transmultiplexer system model, the pulse-shaping wavelet has to be carefully chosen so as to effectively implement the Doppler compensation structure. The desired properties of the pulse-shaping wavelet include 1) compact support/fast decay, 2) sufficient regularity/smoothness in the time domain, 3) PR synthesis/analysis filter bank representation, 4) scale orthogonality, 5) shift orthogonality, and

${ }^{2}$ The concept of rational-homogeneous signals is defined in the sense that they satisfy the rational similarity property with rational scale factor of $1<a<2$, in contrast to the dy-homogeneous signals with dyadic self-similarity property.
6) fine scale resolution. The readers are referred to [7] for a discussion of these desired properties.

A special class of wavelets, the rational orthogonal wavelets is one of the candidates for this application. This class of orthogonal rational wavelets was derived under the framework of a rational multiresolution analysis (MRA) proposed by Auscher in [11] and was generalized by Baussard et al. in [12] to permit more general types of roll-off in the transition bands of the wavelet frequency spectrum. In [7], explicit formulas were derived for the special class of rational wavelets with a dilation factor of $a=(q+1 / q)$ and whose spectrum has no constant passband between the two transition bands.

The rational wavelet function $\psi(t)$ is defined in the frequency domain by

$$
\Psi(\omega)= \begin{cases}(2 \pi)^{-\frac{1}{2}} e^{j \frac{\omega}{2}} \sin \left(\frac{\pi}{2} \beta\left(\frac{q}{\omega_{1}}|\omega|-q\right)\right), & \omega_{1} \leq|\omega| \leq \omega_{2} \\ (2 \pi)^{-\frac{1}{2}} e^{j \frac{\omega}{2}} \cos \left(\frac{\pi}{2} \beta\left(\frac{q}{\omega_{2}}|\omega|-q\right)\right), & \omega_{2} \leq|\omega| \leq \omega_{3} \\ 0, & |\omega| \notin\left[\omega_{1}, \omega_{3}\right]\end{cases}
$$

and the rational scaling function $\phi(t)$ is defined by

$$
\Phi(\omega)= \begin{cases}(2 \pi)^{-\frac{1}{2}}, & |\omega| \leq \omega_{1} \\ (2 \pi)^{-\frac{1}{2}} \cos \left(\frac{\pi}{2} \beta\left(\frac{q}{\omega_{1}}|\omega|-q\right)\right), & \omega_{1} \leq|\omega| \leq \omega_{2} \\ 0, & |\omega|>\omega_{2}\end{cases}
$$

where

$$
\begin{aligned}
& \omega_{1}=\left(q-\frac{q}{2 q+1}\right) \pi \\
& \omega_{2}=a \omega_{1} \\
& \omega_{3}=a \omega_{2}=a^{2} \omega_{1}
\end{aligned}
$$

and $\beta(x)$ is the construction function shaping the spectrum of the transition band, which is not unique. One construction function that leads to desirable decay property of the wavelet is defined by $\beta(x)=x^{4}\left(35-84 x+70 x^{2}-20 x^{3}\right)$. This construction function is adopted in the simulations of this paper. More discussions about the construction functions and other forms of $\beta(x)$ were provided in [12].

Fig. 2 shows the spectrums and waveforms of constructed rational wavelets with dilation factor of $2,3 / 2$, and $4 / 3$, respectively. Notice that as a special case, the $2 / 1$ rational wavelet 
(a)

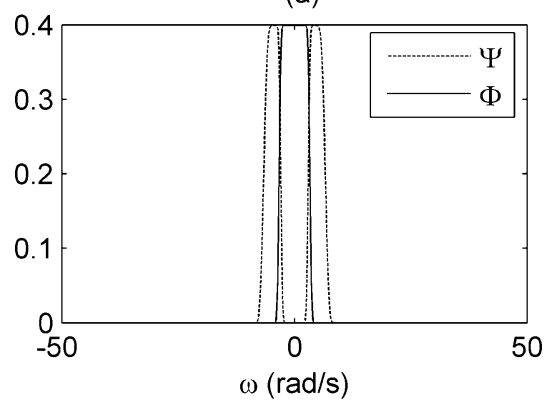

(d)

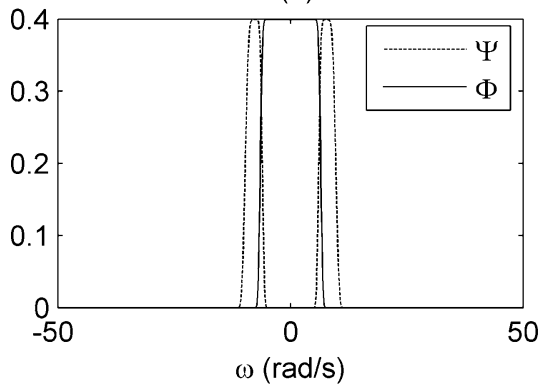

(g)

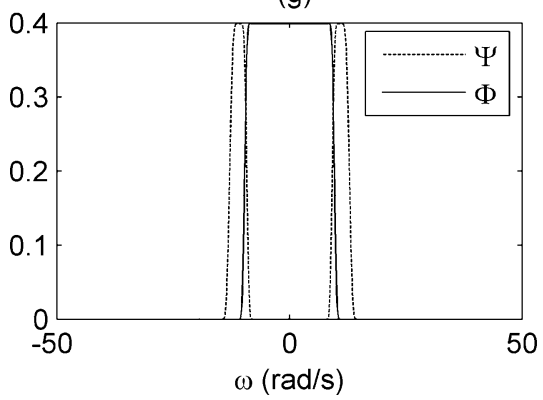

(b)

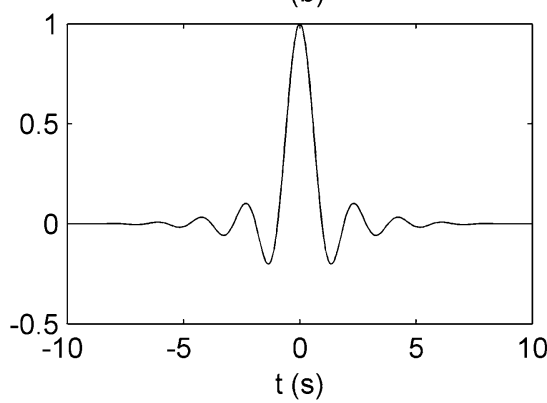

(e)

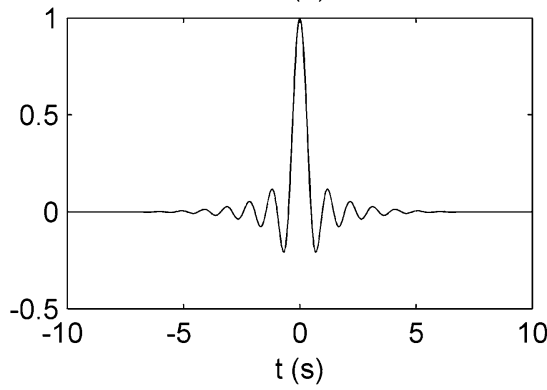

(h)

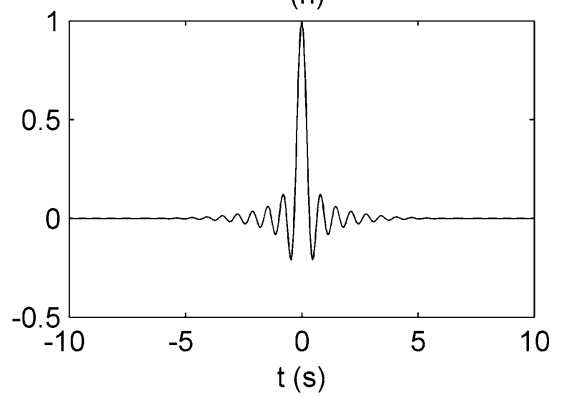

(c)

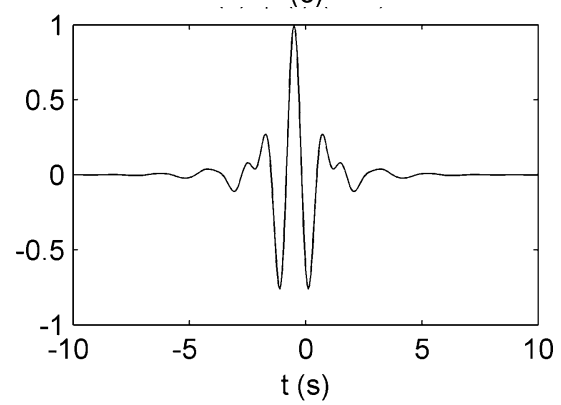

(f)

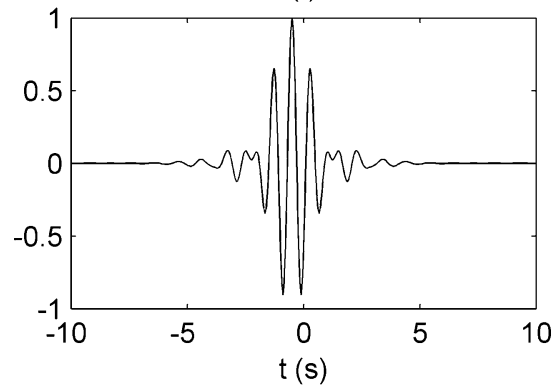

(i)

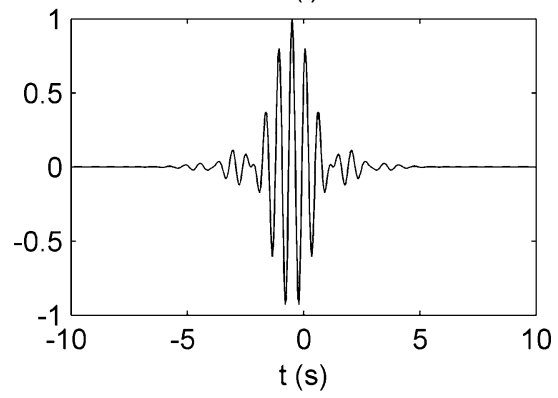

Fig. 2. Several examples of rational orthogonal wavelets. (a) $\Psi(\omega), \Phi(\omega)(a=2) ;$ (b) $\phi(t)(a=2)$; (c) $\psi(t)(a=2) ;($ d) $\Psi(\omega)$, $\Phi(\omega)(a=3 / 2) ;($ e) $\phi(t)(a=$ $3 / 2)$; (f) $\psi(t)(a=3 / 2)$; (g) $\Psi(\omega), \Phi(\omega)(a=4 / 3)$; (h) $\phi(t)(a=4 / 3)$; and (i) $\psi(t)(a=4 / 3)$.

is actually the well-known Meyer wavelet [13]. By the definition in (10), the rational orthogonal wavelets are compactly supported in the frequency domain and therefore have infinite support in the time domain. However, by carefully defining the frequency spectrum, they could have a sufficient decay in the time domain as shown in Fig. 2. This ensures their application as the shaping pulse. Compared with traditional shaping pulses, e.g., the pulses based on raised cosine and square-root raised cosine filters, wavelet-based shaping pulses have unique scale orthogonality and shift orthogonality, which lead to special designs of communication systems, e.g., the Doppler compensation system proposed in this paper and wavelet-based MCM/ OFDM schemes. With respect to the desired properties of the pulse-shaping wavelets for the Doppler compensation structure, there may be other wavelet candidates for this application. The exploration of more wavelet candidates can be treated as another issue, which is beyond the scope of this paper. It should also be noted at this point that scale factors in practical UWA channels are typically quite close to unity, and as such the values of $q$ used in this paper are somewhat unrealistically large, however they permit the design process to be presented in a much simpler manner.

\section{OPtIMUM ReceIVER DeSIGN}

In this section, we design the optimum receivers based on PR wavelet analysis FBs and discuss the characteristics of the receivers that are optimal under the ML decision rule.

\section{A. Equivalence of the Doppler Compensation Structure With an Lth-Order Diversity Communication System}

As shown in (6) and (7) in Section II, with rational orthogonal wavelet pulse signaling, the received signal through the multipath/Doppler channel is a synthesized signal with input wavelet coefficients in certain wavelet subspaces as the delayed, attenuated and periodically zero inserted versions of the symbol sequence $\{s(k)\}$. Equivalently, the receiver is provided with independent fading replicas of the same information-bearing signal. This coincides with the concept of diversity. Compared with the existing diversity techniques, such as frequency diversity, time diversity, and space diversity, the diversity provided by the multiscale nature of the multipath/Doppler channel and rational orthogonal wavelet signaling could be termed as scale diversity in the sense that the same information was carried by multiple signals that are orthogonal in scale. 
Assume there are $L$ dominant paths. The frequency-selective fast-fading multipath/Doppler channels are resolved into $L$ flat slow-fading channels with independent modulation of the same information for each channel. The signals transmitted over the multiple channels are given by

$$
\begin{aligned}
x_{\alpha_{\ell}}(t) & =\sum_{k} s(k) \psi\left(a^{-\alpha_{\ell}} t-k T\right) \\
& =a^{\alpha_{\ell} / 2} \sum_{k} s(k) \psi_{\alpha_{\ell}}\left(t-a^{\alpha_{\ell}} k T\right) .
\end{aligned}
$$

In light of this further insight of the transmultiplexer system model, we see that the addressed wavelet-based Doppler compensation system corresponds to an $L$ th-order diversity communication system. Therefore, the optimum receiver design and the performance characterization also resemble those of an $L$ th-order diversity system. Notice that the same symbol sequence $\{s(k)\}$ is transmitted at different rates $a^{-\alpha_{\ell}} T$ over multiple channels. This is different from conventional diversity systems operating at the same rate on different diversity paths.

\section{B. Optimum Receiver Construction}

We consider optimum receivers for a symbol-by-symbol detection based on the ML criterion. We first recall some facts on the optimum receiver design for a diversity system. Readers are referred to books on communication theory, e.g., [14], for more details, and the notations in [14] are adopted in this section. We use an ML decision rule that is based on the maximization of the likelihood function $P\left(\mathbf{r} \mid \mathbf{s}_{m}\right), m=1,2, \cdots, M$, where $\mathbf{r}=\left[\begin{array}{lll}r_{1} & r_{2} & \ldots \\ r_{N}\end{array}\right]$ is the output observation vector of a correlation or matched filter demodulator, and $N$ is the dimension of the transmitted signal waveforms. The vector $\mathbf{s}_{m}$ is the $M$-ary signal points with components $s_{m k}, k=1,2, \cdots, N$. It is equivalent to finding the signal $\mathbf{s}_{m}$ that maximizes the correlation metrics, as follows:

$$
\begin{aligned}
C\left(\mathbf{r}, \mathbf{s}_{m}\right) & =\underset{ }{2}\left\langle\mathbf{r}, \mathbf{s}_{m}\right\rangle-\left\|\mathbf{s}_{m}\right\|^{2}, \\
& =2 \int_{0}^{T} r(t) s_{m}(t) d t-\varepsilon_{m}, \quad m=1,2, \cdots, M
\end{aligned}
$$

where $r(t)$ is the received signal, $s_{m}(t)$ is the $M$-ary signal waveform, and $\varepsilon_{m}$ is the energy of the signal $s_{m}(t)$. For an $L$ th-order diversity system, the optimum decision is made by maximizing the combined correlation metrics

$$
C M_{m}=\sum_{n=1}^{L} C\left(\mathbf{r}^{(n)}, \mathbf{s}_{m}\right)
$$

where $\mathbf{r}^{(n)}$ is the output observation vector of the $n$th subchannel. Based on the correlation metrics $C\left(\mathbf{r}, \mathbf{s}_{m}\right)$, the optimum receiver can be realized by a correlation or matched filter demodulator followed by symbol detection via selecting the signal corresponding to the largest $C\left(\mathbf{r}, \mathbf{s}_{m}\right)$. Note that the vector $\mathbf{r}$ is reduced to a scalar $r$ for one-dimensional PAM signaling [14].

Returning to the wavelet-based transmultiplexer system model, we see that the PR wavelet analysis FB actually plays

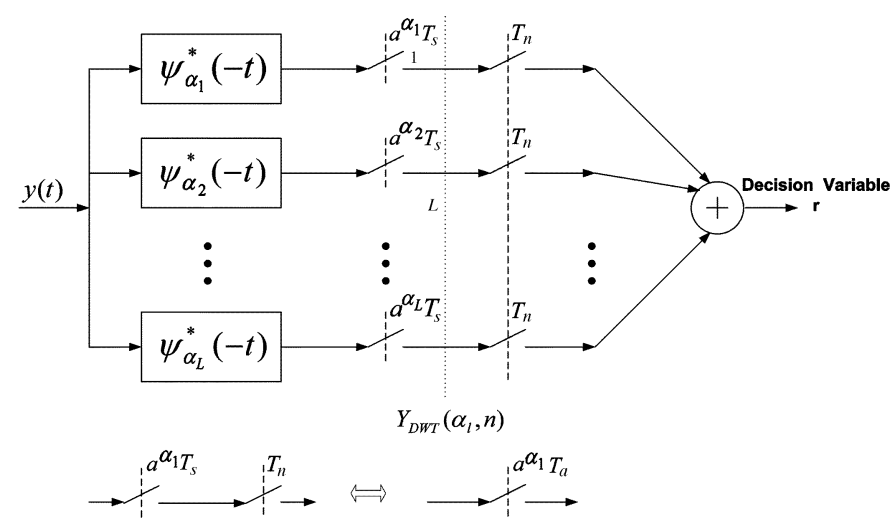

Fig. 3. Type 1 receiver for M-PAM signal.

the role as a optimum multichannel matched filter demodulator (also termed as a Rake receiver) for the transmitted signals $x_{\alpha_{\ell}}(t)=a^{\alpha_{\ell} / 2} \sum_{k} s(k) \psi_{\alpha_{\ell}}\left(t-a^{\alpha_{\ell}} k T_{a}\right)$. The receiver can be regarded as a Rake-type receiver working not only in the time domain in the sense of resolving the multipath, but also in the scale domain in the sense of resolving the Doppler scales. The matched filter $h_{k}(t)$ is related to the wavelet basis functions $\psi(t)$ as $h_{k}(t)=\psi_{k}^{*}(-t)$. Since the wavelet function $\psi(t)$ is real and symmetric as shown in Section II-B, we have $\psi^{*}(-t)=\psi(t)$, which is used to simplify the derivation of related formulas.

We then consider the construction of optimum receivers for $M$-ary PAM wavelet signaling. we see that the multipath/Doppler communication system corresponds to an $L$ th-order diversity system with independent $M$-ary PAM on the waveforms of $\left\{\psi_{\alpha_{\ell}}(t), 1 \leq \ell \leq L\right\}$ over $L$ subchannels. With the PR property of the orthogonal wavelet FB, the optimum receiver can be constructed as shown in Fig. 3. The wavelet coefficients are recovered by sampling at the rate $a^{-k} n T_{s}$. The symbol sequence is then obtained by further downsampling with a sampling factor of $T_{n}$. The combined sampling factor is $a^{-k} T$ as shown in Fig. 3. Although we use a maximum of $(I+J+1)$ channels to capture the information of all possible Doppler scales, we only show the channels corresponding to the existing Doppler scales $\left\{a^{-\alpha_{\ell}}, 1 \leq \ell \leq L\right\}$. These channels have significant energy after filtering while the other channels are eliminated in the receiver diagram.

Note that Fig. 1 illustrates the computation of DWT, not the discrete-time wavelet transform (DTWT) [9], thus the continuous-time sampling factor $a^{-k} n T_{s}$ is allowed for the implementation. For a discrete-time implementation of the receiver, we actually use the truncated discrete-time approximation of the continuous-time wavelet analysis filters and apply a sampling factor according to the length of each analysis filter. For the mathematical convenience, we continue to use the continuous-time wavelet filters in our formulas.

For $M$-ary PAM signaling, a single decision variable suffices, and for the proposed optimum receiver shown in Fig. 3, the decision variable can be derived as

$$
r=\sum_{\ell=1}^{L}\left(a^{\alpha_{\ell} / 2} \rho_{\ell} s_{m} \gamma_{0}+\xi_{\ell}\right)
$$




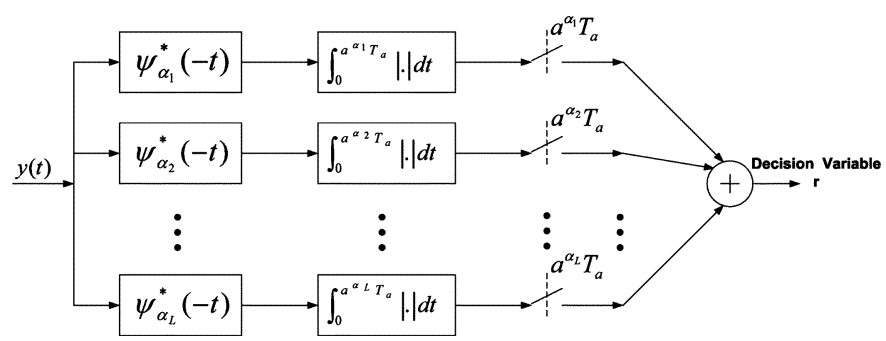

Fig. 4. Type 2 receiver for M-PAM signal.

where

$$
\begin{aligned}
\xi_{\ell} & =\int_{0}^{a^{-\alpha_{\ell}} T} \xi(\tau) \psi_{\alpha_{\ell}}^{*}\left(a^{-\alpha_{\ell}} T-\tau\right) d \tau \\
& =\left.\xi_{\ell}(t)\right|_{t=a^{-\alpha_{\ell}} T} \\
& =\xi_{\ell}\left(a^{-\alpha_{\ell}} T\right)
\end{aligned}
$$

and

$$
\begin{aligned}
\gamma_{0} & =\int_{-\infty}^{\infty} \psi_{\alpha_{\ell}}(\tau) \psi_{\alpha_{\ell}}\left(a^{-\alpha_{\ell}} T-\tau\right) d \tau \\
& =\int_{-\infty}^{\infty} \psi_{\alpha_{\ell}}^{2}(t) d t \\
& =\varepsilon_{\psi_{\alpha_{\ell}}} .
\end{aligned}
$$

$\varepsilon_{\psi_{\alpha_{\ell}}}$ is the energy of the wavelet basis $\psi_{\alpha_{\ell}}$ and $\varepsilon_{\psi_{\alpha_{\ell}}}=1$ for normalized wavelet basis functions. The function $\xi_{\ell}(t)$ is the projection of the AWGN $\xi(t)$ onto the wavelet subspace spanned by $\left\{\psi_{\alpha_{\ell} n}(t), n \in \mathbb{Z}\right\}$.

One problem with this detection algorithm is that it works only with nearly perfect time synchronization. Although the delay factor $\tau_{\ell}^{(1)}$ is resolved as a shift of the output wavelet coefficients, the proposed receiver structure is very sensitive to the estimation of $\tau_{\ell}^{(2)}$ because of the time-variance property of DWT. Furthermore, performance degradation is even worse when the signaling wavelet is a rational wavelet, which is a passband signal, and the outputs of the wavelet analysis FB (actually the autocorrelation function of $\psi_{k}(t)$ ) have severe fluctuations.

Another receiver structure with more robustness against the time synchronization error is shown in Fig. 4. It is based on the optimum envelope detection for signals with random phases [14]. The output decision variable is given by

$$
r=\sum_{\ell=1}^{L}\left(a^{\alpha_{\ell} / 2}\left|\rho_{\ell}\right|\left|s_{m}\right| \mu_{\alpha_{\ell}}+\xi_{\ell}\right)
$$

where

$$
\begin{aligned}
\xi_{\ell} & =\int_{0}^{a^{-\alpha_{\ell}}}\left|\int_{-\infty}^{\infty} \xi(\tau) \psi_{\alpha_{\ell}}^{*}(t-\tau) d \tau\right| d t \\
& =\int_{0}^{a^{-\alpha_{\ell} T}}\left|\xi_{\ell}(t)\right| d t
\end{aligned}
$$

and

$$
\begin{aligned}
\mu_{\alpha_{\ell}} & =\int_{0}^{a^{-\alpha_{\ell}} T}\left|\int_{-\infty}^{\infty} \psi_{\alpha_{\ell}}(\tau) \psi_{\alpha_{\ell}}(t-\tau) d \tau\right| d t \\
& =\int_{0}^{a^{-\alpha_{\ell} T}}\left|\phi_{\psi_{\alpha_{\ell}}} \psi_{\alpha_{\ell}}(t)\right| d t \\
& =a^{\alpha_{\ell}} \mu_{0} .
\end{aligned}
$$

The function $\phi_{\psi_{\alpha_{\ell}} \psi_{\alpha_{\ell}}}(t)$ is the autocorrelation function of $\psi_{\alpha_{\ell}}(t)$ and $\mu_{0}$ is a constant related to the signaling wavelet $\psi(t)$ by $\mu_{0}=\int_{0}^{a^{-\alpha_{\ell} T}}\left|\phi_{\psi \psi}(t)\right| d t$.

We see that, compared with the type 1 receiver, the type 2 receiver is suboptimum in regard to the penalty on the output signal-to-noise ratio (SNR) as illustrated in the next section. The advantage achieved by this detection algorithm is that the requirement on time synchronization is much relaxed. We show in Section IV the performance of the above two receivers denoted as type 1 and type 2 receivers, respectively, by Monte Carlo simulations. The theoretical derivation of probability of error and diversity gain for both receivers is given in Section III-C.

Notice that the type 2 receiver requires positive PAM signaling because of the |.| operation involved in the formula of decision variable (19). Although the type 1 receiver allows standard PAM scheme, positive PAM is adopted for both receivers in the derivation of formulas for a comparison of the system performance.

\section{Evaluation of Probability of Error and Diversity Gain}

To simplify the expression of the signal components out of the analysis FB, we multiply a factor of $a^{-\alpha_{\ell} / 2}$ to the filter in the type 1 receiver, which has the scale factor of $\alpha_{\ell}$, and multiply a factor of $a^{-3 \alpha_{\ell} / 2}$ to the corresponding filter in the type 2 receiver in order to cancel the parameters induced by the different energy of signal waveforms with different scales as shown in (16) and (19). For simplicity, this multiplication is not shown in the receiver block diagrams. The signal points $s_{m}, m=1,2, \cdots, M$ for positive $M$-ary PAM are defined by $s_{m}=A_{m} \sqrt{\varepsilon_{g}}$, where $A_{m}=2 m d, m=1,2, \cdots, M$, and $\varepsilon_{g}$ is the energy of the basic signal pulse $g(t)=\sqrt{\varepsilon_{g}} \psi(t)$. The distance between adjacent signal amplitudes is $2 d$.

For the type 1 receiver, the probability of error can be derived as (see Appendix I)

$$
P_{M}=\frac{2(M-1)}{M} Q\left(\sqrt{\frac{2 d^{2} \varepsilon_{g}\left(\sum_{\ell=1}^{L} \rho_{\ell}\right)^{2}}{N_{0} \sum_{\ell=1}^{L} a^{-\alpha_{\ell}}}}\right) .
$$

This error can be expressed in a simpler form in terms of the average received SNR or output SNR. Assuming $\alpha_{1}<\alpha_{2}<$ $\cdots<\alpha_{L}$, the total symbol rate is equal to the lowest symbol rate through the subchannel with Doppler scaling factor of $a^{-\alpha_{L}}$ and filter length $N_{L}$. Based on this lowest symbol rate, we define 
the average symbol energy of the multipath signal without noise as $\varepsilon_{\mathrm{av}}[14]$ and

$$
\begin{aligned}
\varepsilon_{\mathrm{av}} & =\frac{1}{M} \sum_{m=1}^{M} \varepsilon_{m} \\
& =\frac{d^{2} \varepsilon_{g} a^{\alpha_{L}} \sum_{\ell=1}^{L} \rho_{\ell}^{2}}{M} \sum_{m=1}^{M}(2 m)^{2} \\
& =\frac{2}{3}\left(2 M^{2}+3 M+1\right) d^{2} \varepsilon_{g} a^{\alpha_{L}} \sum_{\ell=1}^{L} \rho_{\ell}^{2} .
\end{aligned}
$$

From (23), we note that

$$
d^{2} \varepsilon_{g}=\frac{3 \varepsilon_{\mathrm{av}}}{2\left(2 M^{2}+3 M+1\right) a^{\alpha_{L}} \sum_{\ell=1}^{L} \rho_{\ell}^{2}} .
$$

By substituting (24) for the $d^{2} \varepsilon_{g}$ in (22), the average probability of a symbol error in terms of the average power is given as

$$
P_{M}=\frac{2(M-1)}{M} Q\left(\sqrt{\frac{3 \gamma_{s} \lambda}{\left(2 M^{2}+3 M+1\right)}}\right)
$$

where $\gamma_{s}=\varepsilon_{\mathrm{av}} / N_{0}$ is defined as the average received SNR per symbol and $\lambda$ is a parameter decided by the multipath attenuation factors and the resolved Doppler scales

$$
\lambda=\frac{\left(\sum_{\ell=1}^{L} \rho_{\ell}\right)^{2}}{a^{\alpha_{L}} \sum_{\ell=1}^{L} \rho_{\ell}^{2} \sum_{\ell=1}^{L} a^{-\alpha_{\ell}}} .
$$

The output SNR obtained by the designed receiver $\gamma_{s, o}$ is

$$
\gamma_{s, o}=\frac{4\left(2 M^{2}+3 M+1\right) d^{2} \varepsilon_{g}\left(\sum_{\ell=1}^{L} \rho_{\ell}\right)^{2}}{3 N_{0} \sum_{\ell=1}^{L} a^{-\alpha_{\ell}}} .
$$

The probability of error can be expressed by $\gamma_{s, o}$ as

$$
P_{M}=\frac{2(M-1)}{M} Q\left(\sqrt{\frac{3 \gamma_{s, o}}{2\left(2 M^{2}+3 M+1\right)}}\right) .
$$

The diversity gain (DG) obtained with noncoherent combining can be expressed as

$$
\mathrm{DG}=10 \log \frac{a^{-\alpha_{\ell}}\left(\sum_{\ell=1}^{L} \rho_{\ell}\right)^{2}}{\rho_{\ell}^{2} \sum_{\ell=1}^{L} a^{-\alpha_{\ell}}} .
$$

For the type 2 receiver, the probability of error is derived as (see Appendix II)

$$
P_{M}=\frac{2(M-1)}{M} Q\left(\sqrt{\frac{2 d^{2} \varepsilon_{g}\left(\sum_{\ell=1}^{L}\left|\rho_{\ell}\right|\right)^{2} \mu_{0}^{2}}{N_{0} \sum_{\ell=1}^{L} a^{-3 \alpha_{\ell}} N_{\ell}}}\right) .
$$

The derivation of $P_{M}$ in terms of the $\gamma_{s}$ and $\gamma_{s, o}$ is similar to the one of the type 1 receiver and is omitted here. There is a performance loss for the type 2 receiver arising from the summation of noise samples, which is evaluated by

$$
P_{l o s s}=10 \log _{10}\left(\frac{\left(\sum_{\ell=1}^{L} \rho_{\ell}\right)^{2} \sum_{\ell=1}^{L} a^{-3 \alpha_{\ell}} N_{\ell}}{\mu_{0}^{2}\left(\sum_{\ell=1}^{L}\left|\rho_{\ell}\right|\right)^{2} \sum_{\ell=1}^{L} a^{-\alpha_{\ell}}}\right)(\mathrm{dB}) .
$$

Given the inequality that $\left(\sum_{\ell=1}^{L} \rho_{\ell}\right)^{2} \leq\left(\sum_{\ell=1}^{L}\left|\rho_{\ell}\right|\right)^{2}$, the $P_{\text {loss }}$ can be bounded by

$$
P_{\text {loss }} \leq 10 \log _{10}\left(\frac{\sum_{\ell=1}^{L} a^{-3 \alpha_{\ell}} N_{\ell}}{\mu_{0}^{2} \sum_{\ell=1}^{L} a^{-\alpha_{\ell}}}\right)
$$

which is independent of the channel attenuation factors.

\section{Simulation Results}

In this section, we present the simulation results addressing the following issues: 1) system discretization, 2) selection of wavelet scale factors, 3) system sensitivity to the time synchronization error, and 4) BER performance versus SNR.

\section{A. System Discretization}

For a discrete-time implementation of the receiver, we use discrete-time approximation of the wavelet basis functions as the analysis filters. In addition, since the rational orthogonal wavelets have infinite support in the time domain, the signaling wavelet has to be truncated. There is a tradeoff among the symbol rate, system complexity, and approximation error in accordance with the sampling rate and the truncation length. For two systems with the same sampling rate and nonoverlapped signaling, the one with a longer truncation length has a better system performance since the frequency spectrum of the wavelet basis functions is better retained; however, it has a longer filter length and slower symbol rate compared with the one of a shorter truncation length. The computation complexity is reduced with a shorter truncation length by applying shorter filters in the receiver; however, more frequency sidelobes are thus induced and result in the interchannel interference (ICI). Note that the "channel" refers to the receiver FB channels with analysis filters orthogonal to each other in an ideal case.

Explicit formulas can be derived to show the relations between these relevant factors. Suppose the signaling pulse is a rational orthogonal wavelet $\psi(t)$ with dilation factor of $a=$ $(q+1 / q)$, which is truncated at $\left[t_{0}, t_{1}\right]$ with $t_{1}-t_{0}=T$. With nonoverlapped signaling, the symbol rate is $f_{I}=1 / T$. From the definition of the rational orthogonal wavelet, we know that the bandwidth (BW) of the wavelet basis function is $\mathrm{BW}_{\psi(t)}=$ $\left[\omega_{1}, \omega_{3}\right]=2 \pi$, where $\omega_{1}=(q-(q / 2 q+1)) \pi$ and $\omega_{3}=a^{2} \omega_{1}$, as shown in (12). Assume the maximum Doppler compression scale is $\hat{a}^{J}$, where $\hat{a}$ is the estimated Doppler dilation factor for a sufficient approximation of the channel Doppler scales. It is worth noting that although we assume $a=\hat{a}$ in the derivation of the transmultiplexer structure in Section II, we can actually select a wavelet with a dilation factor $a$ smaller than the Doppler 
dilation factor $a<\hat{a}$ for a finer resolution. More details about the selection of wavelet dilation factors is given in Section IV-B.

According to the Nyquist sampling principle, to avoid the spectrum distortion, the sample period $T_{s}$ for the signaling pulse has to satisfy

$$
T_{s}=1 / f_{s} \leq \frac{\pi}{\hat{a}^{J} a^{2} \omega_{1}}
$$

The matched filter in the receiver for the scale factor of $\hat{a}^{\alpha_{\ell}}$ will have the length $N_{\ell}$ that

$$
N_{\ell}=\left\lfloor\hat{a}^{\alpha_{\ell}} \frac{T}{T_{s}}\right\rfloor .
$$

By substituting $T_{s}$ of (34) with (33), we have

$$
N_{\ell} \geq\left\lfloor\frac{1}{\pi} \hat{a}^{\left(\alpha_{\ell}+J\right)} T a^{2} \omega_{1}\right\rfloor .
$$

If we keep the same truncated pulse shape and change the symbol rate by letting $f_{I}^{\prime}=C f_{I}$, where $C \in \mathbb{R}$ is a constant, the signaling pulse is actually the dilated wavelet $\psi(C t)$ with a spectrum bandwidth of $\mathrm{BW}_{\psi(C t)}=\left[C \omega_{1}, C \omega_{3}\right]=2 \pi C$. The sample period $T_{s}^{\prime}$ has to satisfy

$$
T_{s}^{\prime} \leq \frac{\pi}{C \hat{a}^{J} a^{2} \omega_{1}}
$$

Suppose we have a positive 4-PAM signaling system with Doppler scales valued at $(3 / 2)^{-1},(3 / 2)^{-2}$ and $(3 / 2)^{-3}$. For simplicity, the attenuation and delay factors are set to be equal for the subchannels in the simulations all through the whole section. The signaling wavelet is the $3 / 2$ wavelet. Therefore, we have $\alpha_{1}=1, \alpha_{2}=2, \alpha_{3}=3, a=\hat{a}=3 / 2$, and $a^{2} \omega_{1}=(8 / 5) \pi$. For this simulation case, since we have known that the Doppler scales are all dilation scales $\left(\alpha_{l}>0\right)$, we can pick the smallest dilation scale $(3 / 2)^{-1}$ to substitute the parameter $\hat{a}^{J}$ in (33). The sampling period $T_{s}$ can then be selected based on the formula (33) (in this case, $T_{s} \leq 5 / 12$ ). Given a truncation length of $T$ and the desired symbol rate $f_{I}^{\prime}$, we can obtain the filter length and the practical sampling rate in the receiver by using formulas (34) and (36).

Based on the above positive 4-PAM signaling system, we examine the impact of discrete-time approximation error that results in the loss of orthogonality (ICI) with the PR transmultiplexer structure. Zero channel noise is assumed. Fig. 5 shows the degree of ICI versus the truncation length $T$. The impact of the sampling rate on the ICI is also shown in Fig. 5. The degree of ICI is evaluated by calculating the normalized standard deviation of the filter output with respect to the ideal output without ICI. In contrast to the one-channel case where the degree of ICI simply represents the effect of truncation and sampling and is calculated to be on the order of $10^{-14}$ or less, Fig. 5 indicates significant performance loss for the multichannel case because of the ICI. However, by minimizing the discretization error, the ICI tends to decrease and the PR condition is approached as derived in the continuous-time context.

It is worth noting that the system discretization has to be considered more carefully for the Doppler compensation system with PSK/QAM signaling where phase information is needed

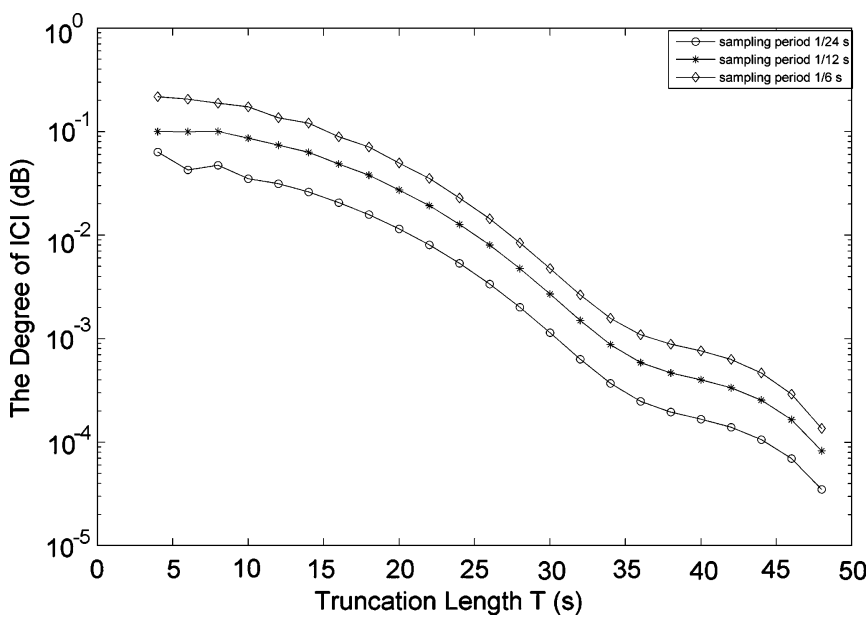

Fig. 5. Degree of ICI versus truncation length $T$ for three-channel model.

and is affected by the length and symmetry of the wavelet filters. In addition, the use of complex rational orthogonal wavelet (CROW) featuring both amplitude and phase information is required [15].

\section{B. Selection of Wavelet Scale Factors}

Besides the discretization effect, the mismatch of the channel Doppler scales and the scales of receiver analysis filters also causes the ICI. In other words, the scale dilation factor of the signaling wavelet has to be selected carefully for a sufficient approximation of the channel Doppler scales. As shown in the formulas of the two decision variables [(16) and (19)], we see that the ICI in the receiver FB is avoided or cancelled due to the orthogonality of the rational wavelet with an ideal matching of the Doppler scales. This is the privilege we obtain by applying the rational orthogonal wavelet as the shaping pulse. This ICI cancellation effect can also be explained from a frequency domain point of view. The signaling with wavelets avoids the relatively large sidelobes which, for example, is a limitation of the DFT-based modulator/demodulator in an OFDM system. The fine scale orthogonality of the rational orthogonal wavelets further ensures the independence of the two signals scaled by close Doppler scales when the spectrum of the two signals is sufficiently apart or orthogonally overlapped.

To illustrate the benefit of using rational orthogonal wavelets instead of the traditional dyadic wavelets as the shaping pulse, we compare the ICI cancellation effect by using dyadic Meyer wavelet signaling and 5/4 rational wavelet signaling respectively. Assuming three existing Doppler scales, $(5 / 4)^{-1}$, $(5 / 4)^{-2}$, and $(5 / 4)^{-3}$, the different ICI cancellation performances are illustrated in Figs. 6-9 by plotting the signal waveforms at different stages of the system. The two simulated systems have the same symbol rate and sample rate. Figs. 6 and 8 show the signals at the synthesis part of the transmultiplexer. Figs. 7 and 9 show the outputs of the analysis FB before multirate sampling and diversity combination (see Fig. 1).

From Fig. 9, we see that with 5/4 rational wavelet signaling, the three Doppler scales can be explicitly resolved with $0-\mathrm{dB}$ noise as shown in (b), (c), and (d), where the information of the symbol sequence is carried by the magnitude of these output 
(a)

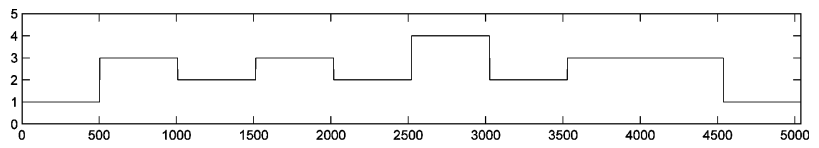

(b)

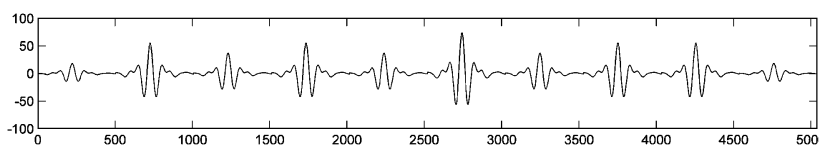

(c)

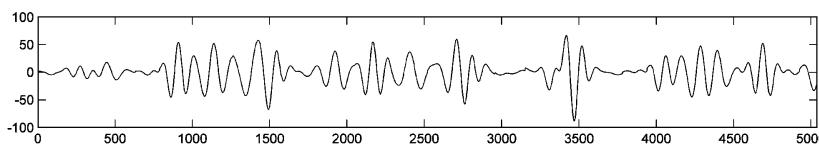

(d)

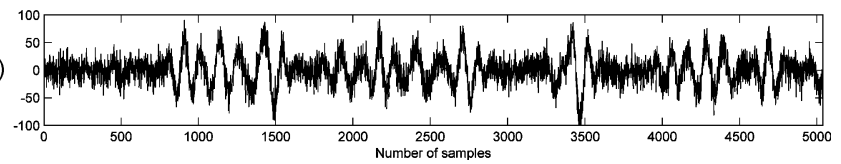

Fig. 6. (a) Input symbol sequence, (b) source signal with Meyer pulse shaping, (c) synthesized multiscale signal with three paths scaled at $(5 / 4)^{-1},(5 / 4)^{-2}$, and $(5 / 4)^{-3}$, and (d) synthesized multiscale signal with 0 -dB AWGN.

(a)

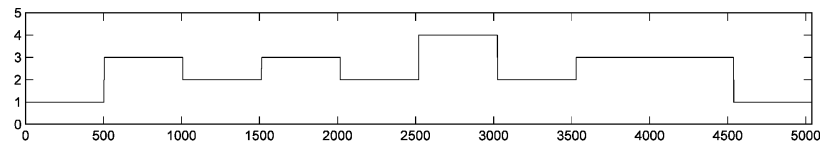

(b)

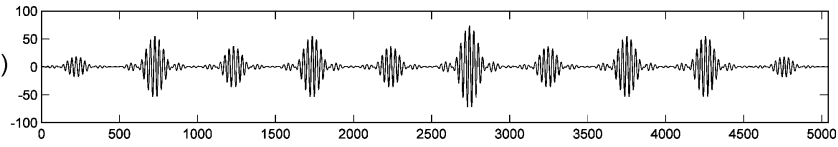

(c)

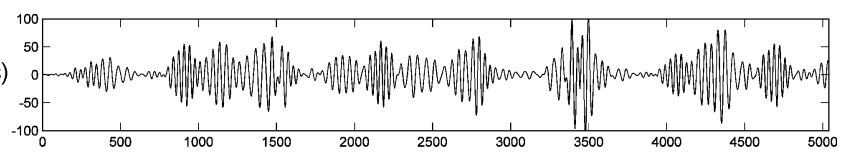

(d)

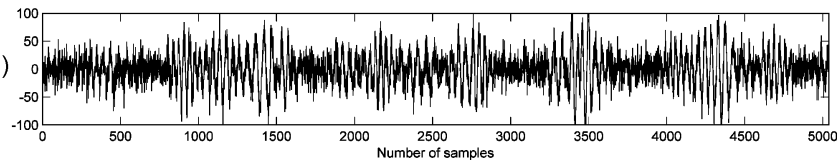

Fig. 8. (a) Input symbol sequence, (b) source signal with rational 5/4 wavelet pulse shaping, (c) synthesized multiscale signal with three paths scaled at $(5 / 4)^{-1},(5 / 4)^{-2}$, and $(5 / 4)^{-3}$, and (d) synthesized multiscale signal with 0 -dB AWGN.

(a)

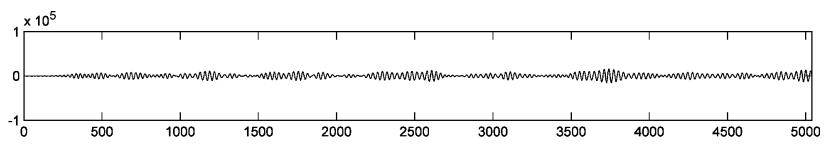

(b)

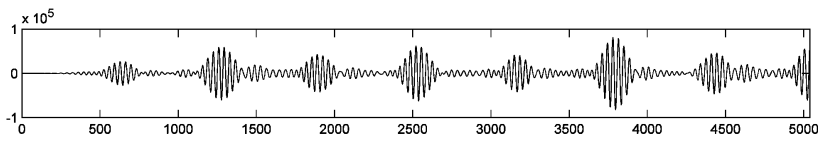

(b)

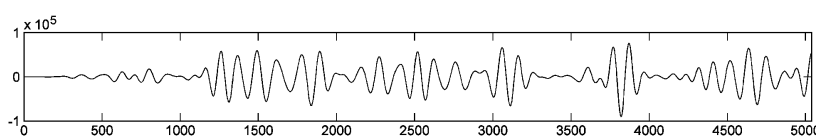

(c)

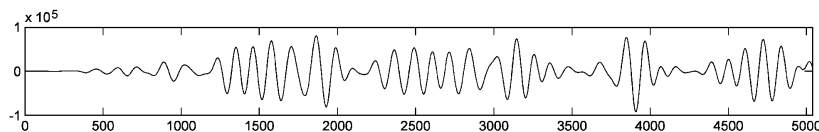

(d)

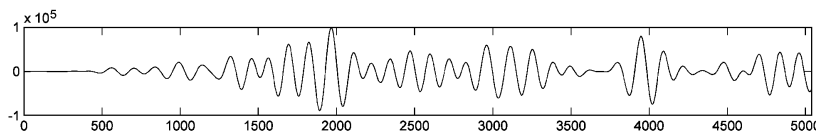

(e)

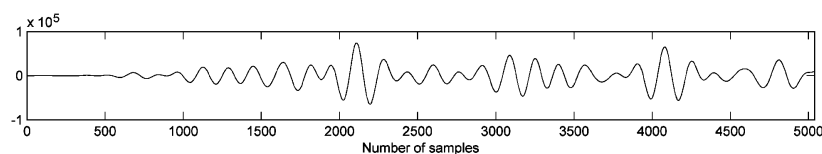

Fig. 7. (a)-(e) Outputs of the analysis filters for Meyer wavelet signaling prescaled at $(5 / 4)^{0},(5 / 4)^{-1},(5 / 4)^{-2},(5 / 4)^{-3}$, and $(5 / 4)^{-4}$, respectively.

pulse sequences. The ICI has been mitigated as shown in (a) and (e). In contrast, with dyadic Meyer wavelet signaling, the three scales cannot be effectively resolved because of the poor ICI cancellation as shown in Fig. 7. Note that the cancellation of ISI caused by the multipath delay shares the same figure of merit with the ICI cancellation based on the Rake-type receiver structure in both time and scale domain as discussed in Section III-B.

Theoretically, with a Doppler scale dilation factor of $\hat{a}$, a rational orthogonal wavelet with a dilation factor of $a=\hat{a}$ suffices due to the scale orthogonality. However, since the rational orthogonal wavelets have infinite support in the time domain, in the practical implementation, a discrete-time approximation is applied, which causes the loss of orthogonality in certain degrees, as shown in Fig. 5. The use of a rational orthogonal wavelet with a dilation factor $a$ smaller than $\hat{a}$ provides a sort of redundancy as illustrated in Fig. 10 and therefore leads to better ICI cancellation. Fig. 10 shows the multipath signal spectrum with $3 / 2$ and $4 / 3$ wavelet signaling, respectively, when the channel has three Doppler scales valued at $(3 / 2)^{-1},(3 / 2)^{-2}$, and $(3 / 2)^{-3}$. Fig. 11 gives a comparison of ICI cancellation for this channel with finer signaling wavelets $(4 / 3,5 / 4$, and $6 / 5$ wavelets) as well as $3 / 2$ wavelet, all with a fixed sampling period of $1 / 6$, and therefore with the same filter length for certain truncation length $T$. We see that the performance of ICI cancellation is improved significantly with finer wavelet scales. The mismatch of the Doppler and wavelet dilation scales, however, may lead to the nonmonotonicity of the performance, which indicates that the ICI may not necessarily be mitigated by the small amount of increase of the truncation length $T$. Again, there is a tradeoff among the wavelet resolution, the symbol rate, the filter 

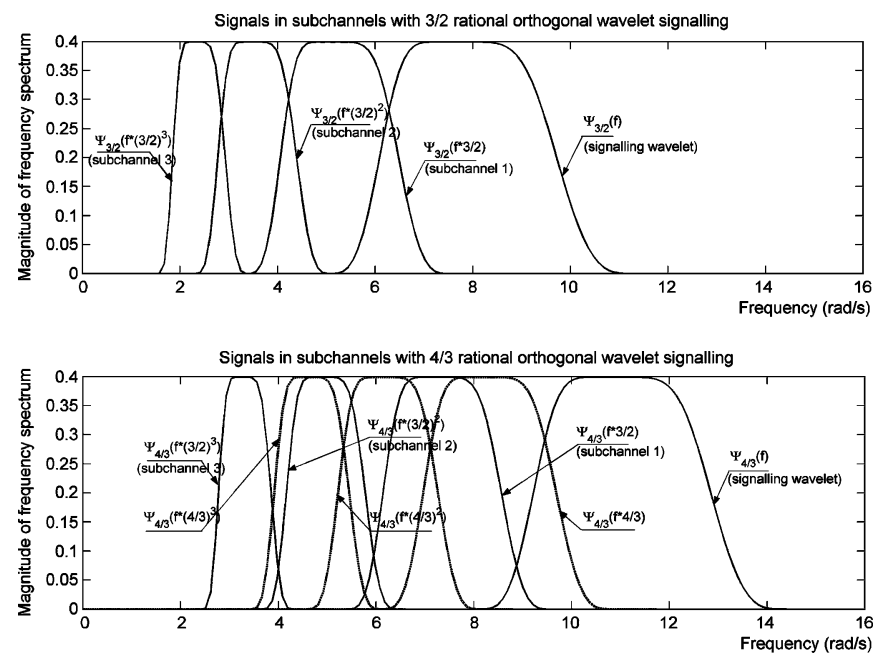

Fig. 10. Frequency spectrum characteristics with $3 / 2$ and $4 / 3$ rational orthogonal wavelet signaling. (The signals with spectrum plotted with the dashed lines are standard orthogonal wavelet functions for $4 / 3$ wavelet, which are shown for a comparison with the signals in the subchannels.)

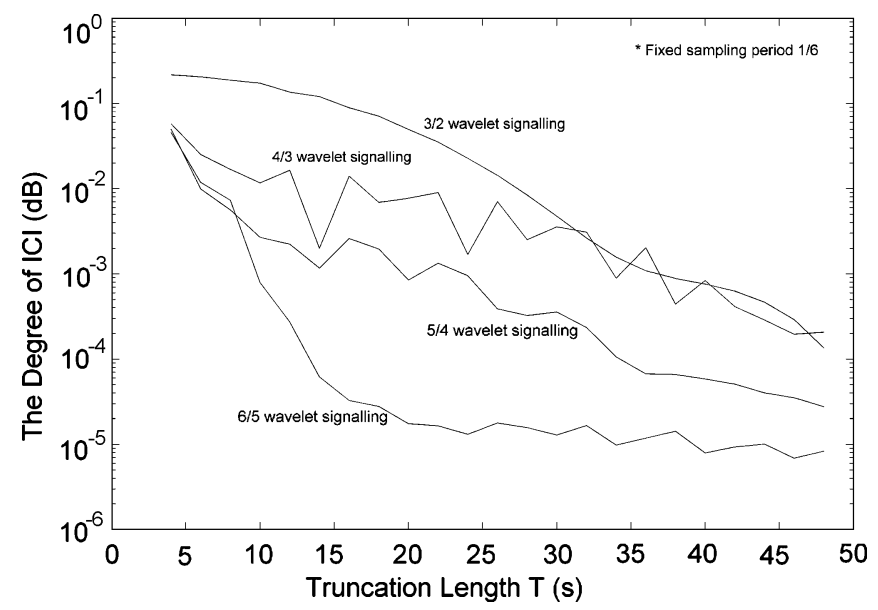

Fig. 11. Degree of ICI versus truncation length $T$ with wavelet dilation factors valued at $3 / 2,4 / 3,5 / 4$, and $6 / 5$, respectively.

length, and the available channel bandwidth, which can be formulated as follows.

Assuming a bandlimited channel with the channel bandwidth $B W=\left[f_{\mathrm{Lo}}, f_{\mathrm{Hi}}\right]$ and Doppler scales $\left\{\hat{a}^{-\alpha_{\ell}}:-J \leq-\alpha_{\ell} \leq\right.$ $I, 1 \leq \ell \leq L\}$, we have

$$
2 \pi f_{\mathrm{Lo}} \leq C \hat{a}^{-I} \omega_{1}<C \hat{a}^{J} \omega_{3} \leq 2 \pi f_{\mathrm{Hi}} .
$$

With the relation in (12), we see that for the existence of a solution for the proposed wavelet signaling scheme, the channel has to meet the condition that $f_{\mathrm{Hi}}>(q+1 / q)^{2} f_{\mathrm{Lo}} \hat{a}^{(I+J)}$, which is satisfied for most of the channels. The formula (37) can be further simplified as

$$
2 \pi f_{\mathrm{Lo}} \hat{a}^{I} \leq C \omega_{1} \leq 2 \pi f_{\mathrm{Hi}} a^{-2} \hat{a}^{-J} .
$$

This constraint on $\omega_{1}$ indicates that the signaling wavelet has to be carefully chosen not only to achieve a sufficient scale resolution but also to satisfy the channel bandwidth requirement.

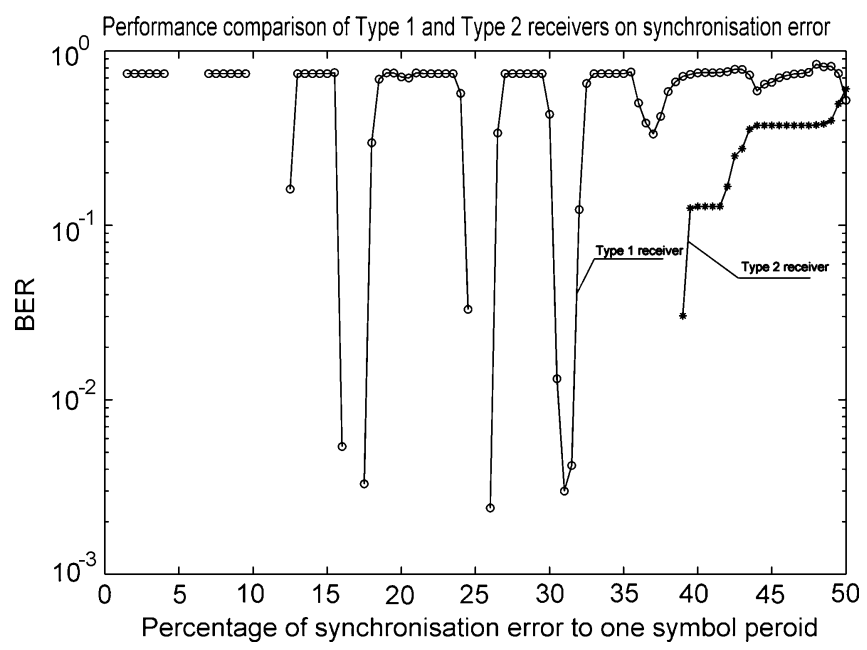

Fig. 12. Performance of two receivers with different synchronization errors with $4 / 3$ wavelet signaling.

Based on a selected signaling wavelet, the maximum symbol rate is derived from (38) as

$$
f_{I, \max }^{\prime}=\frac{2 \pi f_{\mathrm{Hi}}}{a^{2} \omega_{1} \hat{a}^{J} T}
$$

which is restricted by the spectrum characteristics of the selected signaling wavelet $\left(a^{2} \omega_{1}\right)$ and the truncation length $T$.

In general, being aware of the tradeoff between these related system parameters, we have a full control of the system performance by selecting the signaling wavelet (parameter $a$ ) and discretization accuracy (parameters $T_{s}$ and $T$ ) to meet specific system requirements.

\section{System Sensitivity to the Time Synchronization Error}

We simulate a positive 4-PAM signaling system with Doppler scales valued at $(3 / 2)^{-1},(3 / 2)^{-2}$, and $(3 / 2)^{-3}$. The rational orthogonal wavelet with dilation factors of $4 / 3$ is employed as the signaling pulses in the simulation. The matched FB is constructed by prescaling the signaling wavelet according to the estimated Doppler dilation factor which is $3 / 2$ in the simulation. An extension to Doppler scale dilation factors finer than $3 / 2$ is straightforward with rational orthogonal signaling wavelets of finer scale dilation factors and longer analysis and synthesis filters. A randomly generated 100000 symbols are used to test the performance of the proposed receiver structures. Notice that for the limited number of the symbols, the bit error rate (BER) is evaluated to be zero if its value is sufficiently small, which is shown in the subsequent figures on the BER performance.

We compare the sensitivity of the two receivers to the time synchronization error which is illustrated in Fig. 12. The BER is evaluated as a function of synchronization error. Assuming a coarse synchronization is conducted at the input of the receiver, we focus on the synchronization implemented in each subchannel. The synchronization error is denoted as the percentage of maximum timing jitter error out of the symbol rate in each subchannel, and we assume the same error percentage for all subchannels. To exclude the effect of noise on the receiver performance, we set zero AWGN in the analysis. The timing jitter error percentage has a range of $0 \%$ to $50 \%$ with a step 


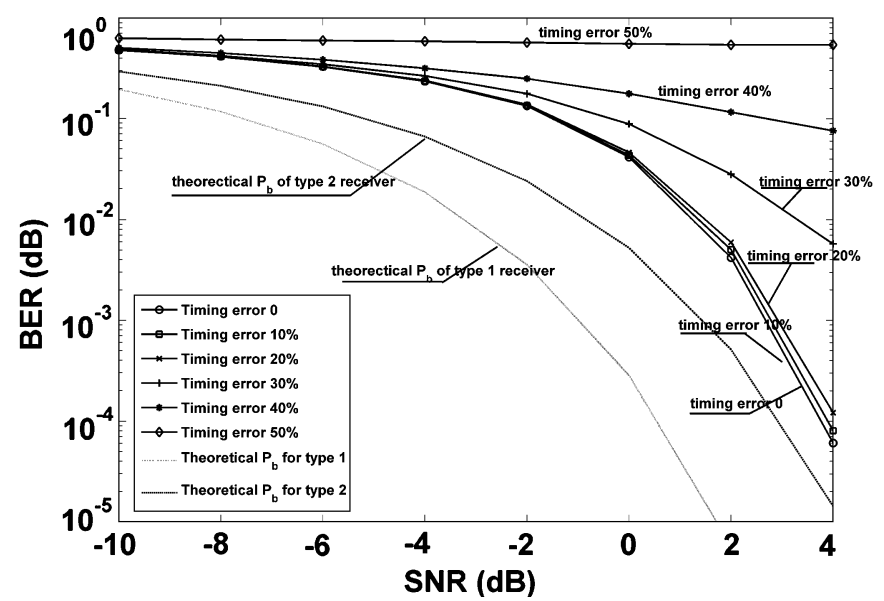

Fig. 13. Performance of type 2 receiver at different SNRs and synchronization errors with $4 / 3$ wavelet signaling.

of $0.5 \%$. For certain timing jitter error percentage, e.g., $30 \%$, the timing error for each symbol is uniformly distributed within $30 \%$ of the symbol period.

As shown in Fig. 12, the type 1 receiver is very sensitive to the synchronization error and can only work with a time error less than $1 \%$. Although good BER shows at certain intervals when the time error is larger than $1 \%$, the receiver becomes inconsistent because of the big fluctuation of the BER performance. A strict time synchronization process is therefore essential for this type of receivers. In contrast, the type 2 receiver shows good adaptation to the synchronization error and works with a time error as large as $40 \%$. The complexity of the receiver structure is significantly reduced with a simple synchronization design (such as the signal energy detection).

\section{BER Performance Versus SNR}

The BER performance of the two types of receivers with different SNR is illustrated in Figs. 13 and 14. For comparison, the SNR performance under different synchronization error with timing error percentage valued at $0 \%, 10 \%, 20 \%, 30 \%$, and $40 \%$ is plotted in the same figure. The SNR is shown as the measured received SNR $\gamma_{m}$, which has the relation with $\gamma_{s}$ (defined in Section III-C) as $\gamma_{m}=\varepsilon_{\mathrm{av}} / N_{0} N_{L}=\gamma_{s} / N_{L}$. In Fig. 13, the type 2 receiver shows good antinoise capability and remains consistent when the timing error is less than 40\%. Fig. 14 shows that for the type 1 receiver the system performance is dominated by the timing error. Moreover, the BER plots with timing error of $2 \%$ and $5 \%$ indicate the fluctuation of the system performance that is in accordance with the results shown in Fig. 12. On the other hand, with a perfect time synchronization, the BER performance of the type 1 receiver outperforms the one of the type 2 receiver. This fact can be evaluated from the computation of the two different decision variables. For the type 2 receiver, the noise term is enhanced in comparison with the type 1 receiver since an integration process on $\xi_{\ell}(t)$ is involved. For both receivers, the simulated system performance is degraded in comparison with the theoretical derivation. This is because of the loss of orthogonality caused by the discrete-time approximation as shown in Section IV-A.

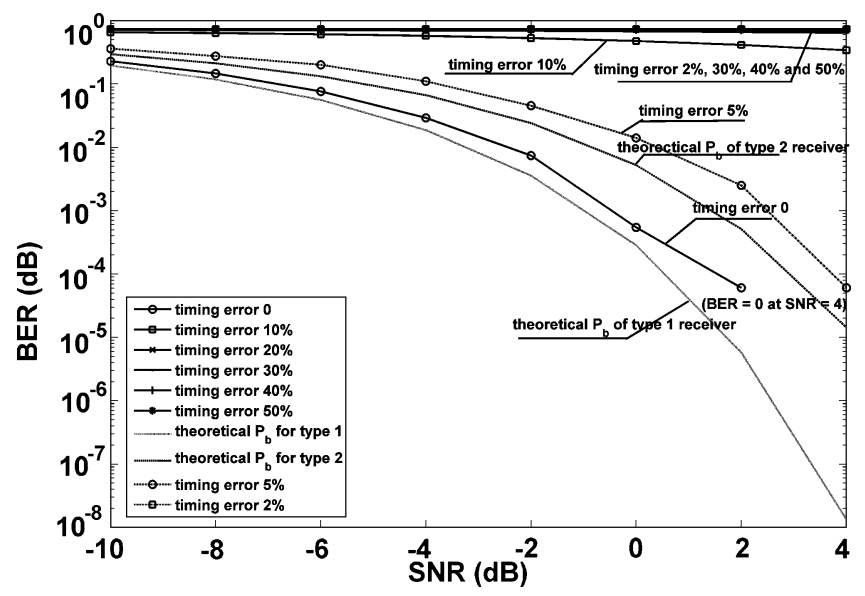

Fig. 14. Performance of type 1 receiver at different SNRs and synchronization errors with $4 / 3$ wavelet signaling.

\section{CONCLUSION}

In this paper, we address the issue of signal transmission and Doppler compensation through multipath/Doppler channels and demonstrate that rational orthogonal wavelet signaling provides a solution that yields an $L$ th-order diversity system. Optimum receivers for the wavelet-based PAM signaling are then designed under the ML decision criterion for diversity combination and symbol detection. Taking advantage of the properties of rational orthogonal wavelets, the designed two types of receivers achieve good system performance in combating the Doppler effect and ISI caused by multipath while mitigating the channel noise. Besides, the type 2 receiver outperforms the type 1 receiver in regard to its unique robustness against the time synchronization error.

Compared with the existing Doppler compensation methods, the proposed broadband Doppler compensation structure leads to less residual Doppler due to the resolving of the multiscale nature. However, the complexity of the system could increase for communication scenarios with slow moving platforms. The selected wavelet dilation factor has to be very small for small Doppler scales to be resolved, which leads to long filters and low symbol rate for a bandlimited channel. Therefore, traditional Doppler compensation schemes are more applicable in this case. The proposed broadband Doppler compensation structure is more suitable for the scenarios when fast moving platforms attempt to communicate. In these cases, the shortage caused by high platform speed for traditional Doppler compensation structures actually becomes an advantage of the proposed system. One specific scenario for a direct application of the proposed system is the communication between underwater vehicles moving at a speed of more than 45 knots. In this case, the magnitude of the Doppler compression or expansion can exceed $1 \%$ and the difference of Doppler scales between eigenpaths reaches $0.1 \%$. The length of filters has to be sufficiently large for this application, which is acceptable for practical implementation due to the low transmission speed of acoustic signals. For the type 1 receiver, more efficient receiver structure can be implemented via a tree-structured FWT algorithm implemented in the frequency domain as shown in 
[12] or by finite-based-response-based approximation as the derivation of the discrete Meyer (dMeyer) wavelet from the Meyer wavelet. However, for the type 2 receiver, the modified receiver structure is based on a parallel filter bank and the advantage it achieves for the synchronization does not hold with a tree-structured filter bank. More efficient implementation may rely on fast Fourier transform (FFT)-based algorithms and can benefit from advanced digital signal processor (DSP) techniques on parallel calculations. Furthermore, the proposed system is also promising for its applications in intersatellite communications and other high-speed aircraft communications.

Notice that to focus on the resolving of the multiscale nature of multipath/Doppler channels, we assume a constant multipath/Doppler channel, which can be resolved into multiple nearly ideal flat fading subchannels, that is, the attenuation and delay coefficients for each multipath are constant for certain subchannel as shown in the channel impulse response $h(\tau)=$ $\sum_{\ell=1}^{L} \rho_{\ell} \delta\left(\tau-\tau_{\ell}\right.$. The designed receiver structure can also be extended to suit more general time-variant channel conditions where the channel impulse response can be expressed as $h(t, \tau)=\sum_{\ell=1}^{L} \rho_{\ell}(t) \delta\left(\tau-\tau_{\ell}(t)\right)$. A multiple-equalizer structure can be implemented straightforwardly with each equalizer adaptive to the impulse response of each subchannel that has been resolved by the designed receiver structure. It has been demonstrated by existing researches [16] that the multiple subchannel equalisers outperform the single channel equalizer in many aspects.

As one of the contributions of this paper, the proposed Doppler compensation structure and its system characterization provides the groundwork for the design of more frequency- efficient communication systems-the MCM or the wavelet-based OFDM communication systems, which utilize rational orthogonal wavelets in different scales as the orthogonal carrier signals. There has been much literature on using dyadic wavelet and wavelet packets for orthogonal multiplexing systems [16]-[19]. The work in this paper that has been focused on the Doppler compensation can be extended straightforwardly to characterize an orthogonal multiplexing system by substituting the resolved Doppler scales with orthogonal carrier scales. One special feature of this orthogonal multiplexing system is the rational orthogonality with scale factor of $1<a<2$. This feature leads to improved spectrum efficiency of the system in comparison with systems based on dyadic orthogonality with $a=2$. More details about the construction and properties of rational orthogonal wavelet-based MCM/OFDM are presented in [15] and [20]. We see that, by letting the intervals of carrier scales greater than the possible channel Doppler spread, and making the scale resolution of the signaling wavelet sufficient for the FB-based Doppler compensation, the rational orthogonal wavelet-based MCM/OFDM communication system provides superior robustness against Doppler dispersion, and could benefit from not only the diversity by orthogonal signaling but also another dimension of diversity provided by the multipath/Doppler channel. Both dimensions of diversity can be explored simultaneously within the same FB receiver structure.

\section{APPENDIX I \\ Derivation of Probability of ERror $P_{M}$ FOR THE TYPE 1 RECEIVER}

The output noise variable is a Gaussian random variable with zero mean and variance

$$
\sigma^{2}=\frac{1}{2} N_{0} \sum_{\ell=1}^{L} a^{-\alpha_{\ell}} .
$$

Assuming that all amplitude levels are equally likely a priori, the average probability of a symbol error is the probability that the noise variable exceeds in magnitude one half of the distance between the received signal levels, which is valued at

$$
d^{\left(\frac{1}{2} e\right)}=d \sqrt{\varepsilon_{g}} \sum_{\ell=1}^{L} \rho_{\ell} .
$$

Taking account of the fact that when either one of the two outside levels is transmitted, an error can occur in one direction, we have

$$
P_{M}=\frac{M-1}{M} \frac{2}{\sqrt{2 \pi} \sigma} \int_{d \sqrt{\varepsilon_{g}} \sum_{\ell=1}^{L} \rho_{\ell}}^{\infty} e^{-\frac{x^{2}}{2 \sigma^{2}}} d x .
$$

By substituting (40) into (42), we have

$$
\begin{aligned}
P_{M} & =\frac{M-1}{M} \frac{2}{\sqrt{2 \pi}} \int_{\frac{d \sqrt{\varepsilon_{g}} \sum_{\ell=1}^{L} \rho_{\ell}}{\sqrt{\frac{1}{2} N_{0} \sum_{\ell=1}^{L} a^{-\alpha_{\ell}}}}}^{\infty} e^{-x^{2} / 2} d x \\
& =\frac{2(M-1)}{M} Q\left(\sqrt{\frac{2 d^{2} \varepsilon_{g}\left(\sum_{\ell=1}^{L} \rho_{\ell}\right)^{2}}{N_{0} \sum_{\ell=1}^{L} a^{-\alpha_{\ell}}}}\right) .
\end{aligned}
$$

APPENDIX II

\section{Derivation of Probability of ERRor $P_{M}$ FOR THE} TYPE 2 RECEIVER

The output noise random variable for the subchannel with Doppler scale of $a^{\alpha_{\ell}}$ can be expressed as

$$
Y=\sum_{i=1}^{N_{\ell}}\left|X_{i}\right|
$$

where $N_{\ell}$ is the filter length and $X_{i}$ is a generalized Rayleigh distributed random variable with one degree of freedom whose probability density function (PDF) is

$$
P(x)=\frac{2}{\sqrt{2 \pi} \sigma} e^{-x^{2} / 2 \sigma^{2}}, \quad x \geq 0 .
$$

The output noise variable $Y$ can be rewritten as $Y=\left|X^{\prime}\right|$, where $X^{\prime}$ is a Gaussian random variable with zero mean and variance $N_{\ell} \sigma^{2}$. Notice that to calculate the tail probability of noise for the type 2 receiver, although the accumulated distribution function (CDF) is single-sided, the PDF of noise is two times the PDF of noise for the type 1 receiver on the positive side. Therefore, for the same noise threshold, the two CDFs are 
equivalent. The probability of error for the type 2 receiver follows the same derivation for the type 1 receiver by substituting the output noise component and signal component of the type 2 receiver.

The output noise variable is equivalent to a Gaussian random variable with zero mean and variance

$$
\sigma^{2}=\frac{1}{2} N_{0} \sum_{\ell=1}^{L} a^{-3 \alpha_{\ell}} N_{\ell}
$$

The one half of the distance between the signal levels is given by

$$
d^{\left(\frac{1}{2} e\right)}=d \sqrt{\varepsilon_{g}} \sum_{\ell=1}^{L}\left|\rho_{\ell}\right| \mu_{0} .
$$

The probability of error is derived as

$$
P_{M}=\frac{2(M-1)}{M} Q\left(\sqrt{\frac{2 d^{2} \varepsilon_{g}\left(\sum_{\ell=1}^{L}\left|\rho_{\ell}\right|\right)^{2} \mu_{0}^{2}}{N_{0} \sum_{\ell=1}^{L} a^{-3 \alpha_{\ell}} N_{\ell}}}\right) .
$$

\section{ACKNOWLEDGMENT}

The authors would like to thank the anonymous reviewers and the Associate Editor Dr. Timothy N. Davidson for their constructive comments to improve the quality of this manuscript.

\section{REFERENCES}

[1] T. S. Rappaport, Wireless Communications: Principles and Practise, 2nd ed. Englewood Cliffs, NJ: Prentice-Hall, 2002.

[2] Y. V. Zakharov and V. P. Kodanev, "Multipath-doppler diversity of OFDM signals in an underwater acoustic channel," in Proc. IEEE Conf. Acoustics, Speech, Signal Processing (ICASSP), Jun. 2000, vol. 5, pp. 2941-2944.

[3] J. G. Proakis, M. Stojanovic, and J. A. Catipovic, "Adaptive equalization algorithms for high rate underwater acoustic communications," in Proc. 1994 Symp. Underwater Vehicular Technology (AUV'94), 1994, pp. $157-164$.

[4] A. Benson, J. Proakis, and M. Stojanovic, "Towards robust adaptive acoustic communications," in Proc. MTS/IEEE OCEANS'00 Conf., Sep. 2000, vol. 2, pp. 1243-1249.

[5] B. S. Sharif, J. Neasham, O. R. Hinton, and A. E. Adams, "A computationally efficient Doppler compensation system for underwater acoustic communications," IEEE J. Ocean. Eng., vol. 25, no. 1, pp. 52-61, Jan. 2001.

[6] M. Johnson, L. Freitag, and M. Stojanovic, "Improved Doppler tracking and correction for underwater acoustic communications," in Proc. IEEE Conf. Acoustics, Speech, Signal Processing (ICASSP), 1997, vol. 1, pp. 575-578.

[7] L. Yu and L. B. White, "Broadband Doppler compensation for rational wavelet-based UWA communication systems," in Proc. Asia-Pacific Conf. Communications (APCC), 2005, pp. 605-609.

[8] W. C. Jakes, Microwave Mobile Communications. New York: Wiley, 1974.

[9] P. P. Vaidyanathan, Multirate Systems and Filter Banks. Englewood Cliffs, NJ: Prentice-Hall, 1993.

[10] G. W. Wornell and A. V. Oppenheim, "Wavelet-based representations for a class of self-similar signals with application to fractal modulation," IEEE Trans. Inf. Theory, vol. 38, no. 2, pp. 785-800, Mar. 1992.
[11] P. Auscher, "Wavelet bases for L2(R) with rational dilation factor," in Wavelets and Their Applications. Boston, MA: Jones \& Barlett, 1992, pp. 439-451.

[12] A. Baussard, F. Nicolier, and F. Trucheter, "Rational multiresolution analysis and fast wavelet transform: Application to wavelet shrinkage denoising," Signal Process., vol. 84, no. 10, pp. 1735-1747, 2004.

[13] W. W. Jones and J. C. Dill, "The square root raised cosine wavelet and its relation to the Meyer wavelet," IEEE Trans. Signal Process., vol. 49, no. 1, pp. 248-251, Jan. 2001.

[14] J. G. Proakis, Digital Communications, 5th ed. New York: McGrawHill, 2001.

[15] L. Yu and L. B. White, "Complex rational orthogonal wavelet and its application in communications," IEEE Signal Process. Lett., vol. 13, no. 8, pp. 477-480, Aug. 2006.

[16] S. Srinidhi, J. G. Proakis, and M. Stojanovic, "Wavelet based modulation for frequency hopped spread spectrum communications," in Proc. IEEE Vehicular Technology Conf. (VTC), 1999, vol. 2, pp. 904-908.

[17] M. Luise, M. Marselli, and R. Reggiannini, "Symbol timing recovery for wavelet-based linear modulation," in Proc. IEEE Global Telecommunications Conf. (IEEE GLOBECOM), 1999, pp. 2513-2517.

[18] A. R. Lindsey, "Wavelet packet modulation for orthogonally multiplexed communication," IEEE Trans. Signal Process., vol. 45, no. 5, pp. 1336-1339, May 1997.

[19] K. M. Wong, J. Wu, T. N. Davidson, and Q. Jin, "Wavelet packet division multiplexing and wavelet packet design under timing error effects," IEEE Trans. Signal Process., vol. 45, no. 12, pp. 2866-2890, Dec. 1997.

[20] L. Yu and L. B. White, "A new OFDM scheme based on complex rational orthogonal wavelets," presented at the Defence Appl. Signal Process. Workshop (DASP'2006), Queensland, Australia, Dec. 10-14, 2006.

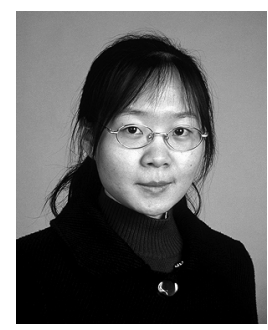

Limin Yu received the B.Eng. degree in telecommunications engineering and the M.Sc. degree in radio physics/underwater acoustic communications from Xiamen University, Xiamen, China, in 1999 and 2002 , respectively. She is currently working towards the Ph.D. degree at the Department of Electrical and Electronic Engineering, The University of Adelaide, Adelaide, Australia.

From 1999 to 2002, she was with the National Key Laboratory of Underwater Acoustic Communications in Xiamen University. From 2002 to 2003, she worked at the 3G Development Group, Software Support Division, ZTE Telecommunication Company, Limited, Shenzhen, China. Her research interests include wavelet, multirate filer banks, and signal processing theory with applications in underwater acoustic communications and wireless communication networks.

Ms. Yu received the Xinhua Science and Technology Award in 1997 and 1998, respectively, and the Huawei Award in 2000 from Xiamen University. She is a recipient of IPRS scholarship from the Australian government.

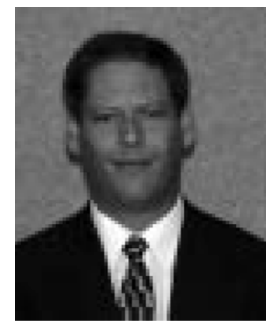

Langford B. White (SM'00) received the B.Sc. (Math.), B.E. (Hons.), and Ph.D. (Elect. Eng.) degrees from the University of Queensland, Brisbane, Australia, in 1984, 1985, and 1989, respectively.

From 1986 to 1999, he worked for the Defence Science and Technology Organization, Salisbury, Australia. Since 1999, he has been a Professor with the School of Electrical and Electronic Engineering, The University of Adelaide, Adelaide, Australia, where he is also Director of the Centre for Internet Research His research interests include signal processing, control, telecommunications, and Internet engineering. 